\title{
Facial Recognition Utilizing Patch Based Game Theory
}

\author{
Foysal Ahmad, Kaushik Roy, Brian O'Connor, Joseph Shelton, Pablo Arias, Albert Esterline, and Gerry \\ Dozier
}

\begin{abstract}
This paper presents an efficient algorithm for face recognition using game theory. Texture based feature extraction techniques are popular for facial recognition, specifically those that segment a facial image into even sized regions, or patches. A cooperative game theory (CGT) based patch selector is exploited to select the most salient patches to extract features. The patches that have a stronger individual importance along with a strong interaction with other patches are selected. A modified local binary pattern (mLBP) feature extraction technique is utilized to extract features from each patch. The performance of the proposed scheme is validated using the Face Recognition Technology (FERET) database. Results show that compared to using mLBP alone, the CGT based selector outperforms it in regards to accuracy and amount of pathces used among different patch resolutions.
\end{abstract}

Index Terms-Face recognition, modified local binary pattern (mlbp), game theory, and patch selection.

\section{INTRODUCTION}

Face recognition technology has received significant attention in the past several years due to its potential for a wide variety of applications in both law enforcement and non-law enforcement. Current face recognition algorithms usually rely on a very good initial alignment and illumination of the faces to be considered for performance evaluation to ensure the higher performance. Under controlled image acquiring constraints, it is possible to capture high quality images and achieve an impressive accuracy with a very low error rate. However, illumination invariance, facial expressions, and partial occlusions are some of the most challenging problems in face recognition and decrease recognition accuracies substantially. Several researchers proposed different facial recognition algorithms to tackle these problems [1]-[3]. The face recognition algorithms based on local appearance descriptors such as Gabor filters, SURF, SIFT, and histograms local binary patterns (LBP) provide more robust performance against occlusions, different facial expressions, and pose variations than the holistic approaches [4], [5]. The LBP-based feature extractor has proven to be highly distinctive and its key advantages includes robustness against illumination and pose variations [6]. The LBP operator was employed to extract the discriminative facial features [4], [5]. In this paper, we apply a modified LBP (mLBP), which fuses both the sign and

Manuscript received November 19, 2014; revised February 26, 2015 This work was supported in part by Science and Technology Center: Bio/Computation Evolution in Action Consortium (BEACON).

The authors are with the North Carolina A\&T State University, Greensboro, NC 27411 USA (e-mail: fahmad@aggies.ncat.edu, kroy@ncat.edu, bpoconno@aggies.ncat.edu, jashelt1@aggies.ncat.edu, parias@aggies.ncat.edu, esterlin@ncat.edu,gvdozier@ncat.edu). magnitude features, to improve the facial texture classification performance [7]. Though the sign component of LBP operator preserves most of the information of local difference, the magnitude component provides additional discriminant information that enhances the overall recognition accuracy.

In machine learning, selecting the best features from the higher-dimensional feature space has several potential benefits, including confronting the problem of dimensionality to enhance the prediction performance, reducing the measurement and storage requirements and decreasing the training and prediction times [8]. Several different approaches are conducted in feature selection such as adaboost [9], genetic algorithms [10], simulated annealing [11], SVM [12], and boosting method [13]. Most of these selectors ignore features which as a group have strong discriminatory power but are individually weak [14]. To handle this problem, a Coalition Game Theory (CGT) model is utilized to select only important patches over entire image area instead of selecting only individual features. By selecting only the important patches instead of the entire image, the recognition process of face images can focus only on those patches and hence reduce overall complexity and time. The CGT evaluates each patch according to its influence to the intricate and intrinsic interrelations among patches based on Shapley value [15]. Each patch performs as a player in this model and the patches with most contribution in the coalition's outcome are selected [16]. Previously we applied similar approach for selecting patches in iris recognition process [16].

The remainder of this paper is organized as follows. Section II provides an overview of feature extraction. Section III describes the feature selection technique using coalition game theory. In Section IV, we present our experimental results. Finally, Section V provides the conclusions.

\section{Feature Extraction Using MLBP}

The LBP method was first proposed by Ojala et al. [6] to encode the pixel-wise information in images. Images are probed locally by sampling grayscale values at a central point $g_{c}$ and $P$ points at $g_{1}, g_{2} \ldots . . g_{p-l}$ spaced equidistantly around a circle of radius $R$.

$$
L B P_{P, R}=\sum_{p=0}^{p-1} r\left(g_{p}-g_{c}\right) 2^{p}, r(x)=\left\{\begin{array}{l}
1, x \geq 0 \\
0, x<0
\end{array}\right.
$$

where $g_{c}$ denotes the gray level value of the center pixel, $g_{p}$ represents the value of the neighboring pixels of the center, $P$ is the total number of neighboring pixels and $\mathrm{R}$ is 
the radius of the neighborhood. For an image of size $I^{\star} J_{x}$ LBP pattern is computed for each pixel of an image and a histogram is developed to represent the face texture:

$$
\begin{gathered}
\operatorname{Hist}(k)=\sum_{i=1}^{I} \sum_{j=1}^{J} f\left(L B P_{P, R}(i, j), k\right), k \in[0, K] \\
f(x, y)=\left\{\begin{array}{l}
1, x=y \\
0, \text { otherwise }
\end{array}\right.
\end{gathered}
$$

where $K$ denotes the maximal LBP pattern value. Now, we calculate the local difference, Dist $_{\mathrm{p}}$, between the center pixel $g_{c}$ and the evenly spaced neighboring pixels, $\mathrm{g}_{\mathrm{p}}, p=0,1,2 \ldots$ $P-1$ as Dist $_{p}=g_{p}-g_{c}$. Thus, we obtain the image local structure at $g_{c}$ with the local difference vector $\left[\right.$ Dist $_{0}, \ldots$, Dist $\left.t_{p-1}\right]$. Since the center intensity value, $g_{c}$ is removed, the local difference vector provides robust performance against the illumination changes. In addition to the traditional LBP implementation, the mLBP technique also incorporates another component. We decompose the Dist $t_{p}$ into two components [7]:

$$
\operatorname{Dist}_{p}=s_{p} \times m_{p}
$$

where $S_{p}=\left\{\begin{array}{l}1, \text { Dist }_{p} \geq 0 \\ -1, \text { Dist }_{p}<0\end{array}\right.$ and $m_{p}$ are the sign and magnitude of Dist $_{p}$, respectively.
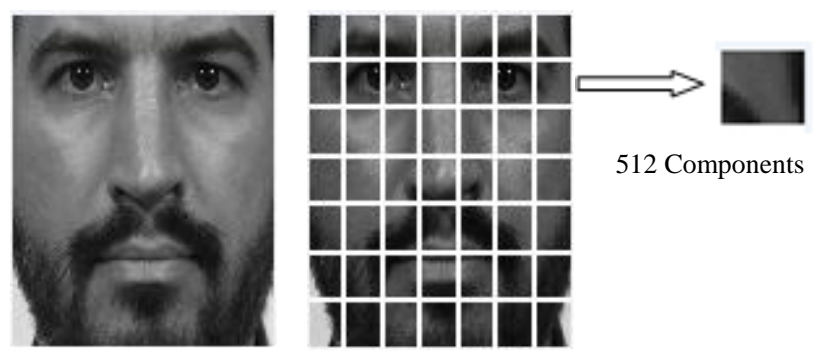

(a) Frontal view
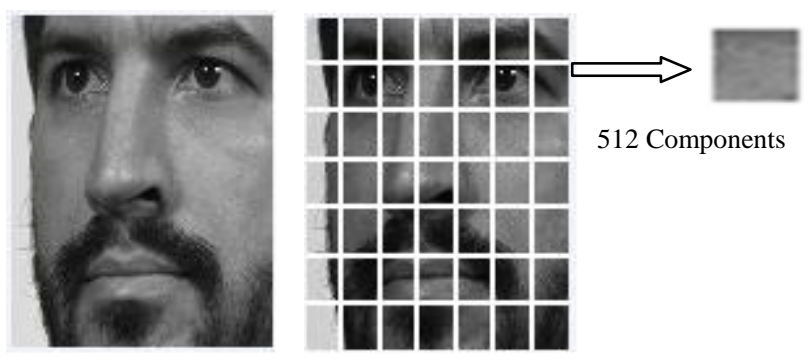

(b) Angle view
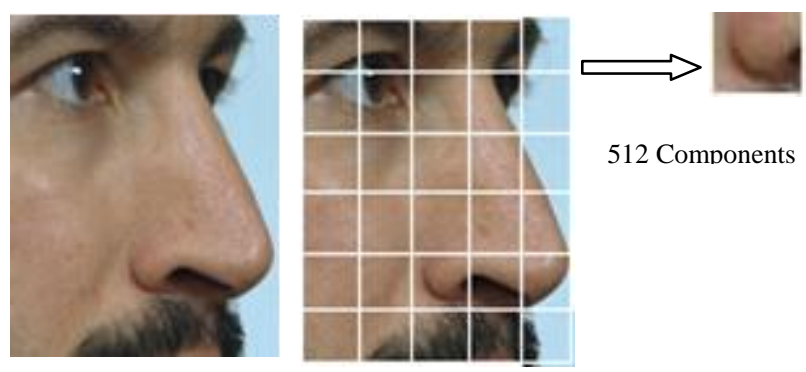

(c) Half right

Fig. 1. Facial images with different patches.
In this paper, we utilize the complementary strengths of the sign and magnitude components of Dist $_{p}$ in order to improve the texture classification performance. The equation (3) represents the local difference Sign Magnitude Transform (SMT). To extract the facial textural features, first, we divide the facial image into several patches and apply the mLBP on each patch (see Fig. 1). Each facial patch is represented by 256 sign and 256 magnitude components. We concatenate the sign and magnitude components and present a single patch by $256 \times 2=512$ components. Depending on the number of patches created, the overall features obtained can be dramatically large. For example, a subject's image split into $4 \times 6$ patches results in 24 patches. Each one of these patches has 512 features, leading to 12,288 features to represent the facial image.

To determine the identification accuracy of a particular feature extraction technique on a dataset of facial subjects, the subject instances are divided into a probe set and a gallery set. The probe set represents users attempting to gain access into a system, while the galley set represents a database of enrolled subjects. All instances are converted into FVs using the feature extraction technique. Some distance metric such as Euclidean distance or Manhattan distance is used to determine the similarity between probe instances and gallery instances. The gallery instance with the smallest distance will be considered the match. The accuracy of the feature extractor depends on how many probe instances are correctly matched with their corresponding gallery instances.

\section{Patch Selection Using CGT}

CGT is concerned with situations in which the decision-makers interact with one another and the reward for each participant in the coalition depends not just on his own decisions but on the decisions made by everyone. Coalition games involve a set of players and a reward associated with different groups or coalitions of players. The reward of a certain coalition depends on individual contributions of players composing this coalition to the game. The larger the contribution of a player is, the higher the benefit of having this player in a coalition. Coalitions with high reward are naturally preferable over those with small reward. This perspective yields an iterative algorithm, contribution selection algorithm (CSA), for patch selection to optimize the performance of the classifier on unseen data [17]. In this approach, each patch obtained using mLBP extractor is regarded as player. The $\mathrm{mLBP}$ technique fuses both the sign and magnitude feature components to improve the feature extraction performance; whereas the traditional LBP utilizes only the sign information. Even though the sign component of LBP operator preserves most of the information of local differences, the magnitude component provides additional discerning features that can improve the overall classification accuracy. The CSA algorithm ranks each patch on each step by using the classifier. Previously we applied this patch selection algorithm on iris recognition [16]. Here the application of CGT is applied for face recognition. The ranking is based on the Shapley value reported in [15], a well-known concept from game theory, to estimate the 
importance of each patch considering the interactions between patches [18].

Shapley values measures the distribution of the power among the players in the voting game, which can be transformed into the arena of patch/feature selection attempting to estimate the importance of each patch. The idea is motivated by the observation that every subset of patches can be regarded as a candidate subset for the final selected optimal subset and the power of each patch can be measured by averaging the contributions that it makes to each of the subset which it belongs to.

The Shapley value is defined as follows [15]. Let the marginal importance of player $i$ to a coalition $S(i \notin S)$ be:

$$
\Delta_{i}=v(S \cup\{i\})-v(S)
$$

where $v(S)$ is the reward associated with coalition $S$. The reward can be negative, zero, or positive. The negative or zero reward implies no benefits of inclusion of player $i$ into the current coalition. The Shapley value is then defined as:

$$
\Phi_{i}(v)=\frac{1}{\text { factorial }(n)} \sum_{\pi \in \Pi} \Delta_{i}\left(S_{i}(\pi)\right)
$$

where $n$ is the total number of players, $S_{i}(\pi)$ is the set of players appearing before player $i$ in permutation $\pi$, and $\Pi$ is the set of permutations over $n$. Thus, the Shapley value of a given player is the mean of its marginal importance averaged over all possible coalitions of players.

In this research effort, we apply the forward selection algorithm. The CSA iteratively selects patches through forward selection. The forward selection variant iteratively adds a predefined number of patches with the highest contribution to the classification accuracy as long as there are patches with sufficiently large contribution values exceeding a preset contribution threshold. This algorithm returns a contribution value for each patch according to its role in enhancing the classifier's performance considering its interaction with other patches.

\section{EXPERIMENTAL RESULTS}

In this research effort, the Face Recognition Technology (FERET) program database [19] is used for experiments. The FERET database was collected in 15 sessions between August 1993 and July 1996. FERET has roughly 5138 facial images from 723 subjects and the instances per subject vary from 2 to 60 instances. In our experiments, 4415 images are placed in the gallery set and 723 images are placed in the probe set. In FERET dataset, many instances from each subject are affected by different non-ideal factors, including subject's alignment to the camera, facial expression, gaze deviation, and facial hair styling. Therefore, these noise factors make it difficult to produce accurate results. Fig. 2 shows some samples of facial images that have been preprocessed by cropping. As mentioned in Section II, we divide each subject's image into a multitude of different patch variations and generate $\mathrm{mLBP}$ feature vectors for each patch. While each LBP patch provides 256 features (only sign components), each mLBP patch produces 512 features as it utilizes both the sign and magnitude components for experiments. We conduct an extensive set of experiments to evaluate the performance in two modes: verification (one-to-one) and identification (one-to-many). In the verification mode, we measure the performance in terms of Genuine Accept Rate (GAR) and False Accept Rate (FAR) with the assumption that a test sample comes from a specific subject. In the identification mode, we make a one-to-many search in the entire dataset for a given test sample to find the best matched sample. The one-to-many matching performance results are enlisted in Table I. The first column of Table I includes different patch combinations, accuracy with only mLBP and accuracy with CGT and mLBP are enlisted in column 2 and column 3 respectively. The percentages of selected patches of total patches are included in parentheses in column 3. It is shown in Table I that CGT model improved the recognition performance using a smaller image area to evaluate the performance. Among different combinations of patches, $5 \times 5$ combinations provided best matching results using only $40 \%$ of entire image area. Further analysis is shown using the Cumulative Match Characteristics (CMC) curve and Receiver Operator Characteristics (ROC) curve only for important and selected patches using CGT model and mLBP patch extractor. Fig. 3 depicts the ROC performance for face recognition and CMC curve is shown in Fig. 4. From Fig. 3, it can be shown that the GAR is $33.5 \%$ at $1 \%$ FAR.

TABLE I: FACE RECOGNITION MATCHING RESULTS

\begin{tabular}{|c|c|c|}
\hline $\begin{array}{c}\text { Patches } \\
(R \times C)\end{array}$ & $\begin{array}{c}\text { Accuracy with } \\
\text { mLBP }\end{array}$ & $\begin{array}{c}\text { Accuracy with mLBP and CGT (\% } \\
\text { area used) }\end{array}$ \\
\hline $4 \times 6$ & 0.8546 & $0.8837(41 \%)$ \\
\hline $5 \times 5$ & 0.8518 & $0.9086(40 \%)$ \\
\hline $5 \times 6$ & 0.8601 & $0.8961(33 \%)$ \\
\hline $6 \times 6$ & 0.8629 & $0.8878(27 \%)$ \\
\hline $6 \times 7$ & 0.8407 & $0.8684(35 \%)$ \\
\hline $8 \times 5$ & 0.8463 & $0.8961(37 \%)$ \\
\hline $8 \times 6$ & 0.8560 & $0.8864(41 \%)$ \\
\hline $8 \times 8$ & 0.831 & $0.8712(62 \%)$ \\
\hline $10 \times 6$ & 0.8740 & $0.8961(50 \%)$ \\
\hline $10 \times 10$ & 0.838 & $0.8975(25 \%)$ \\
\hline
\end{tabular}

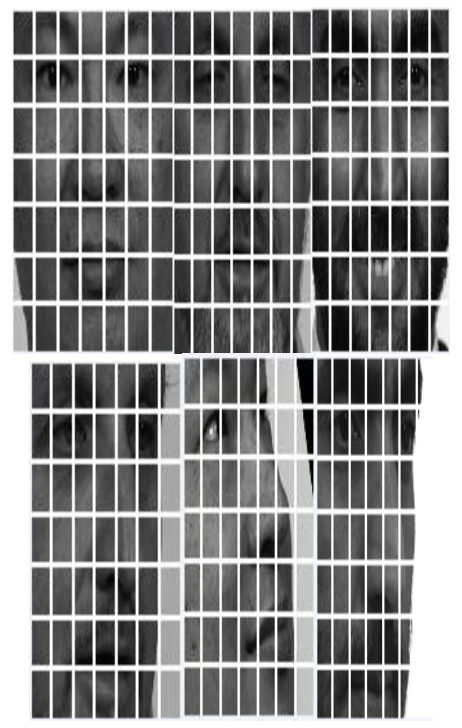

Fig. 2. Facial image samples with patches from FERET. 


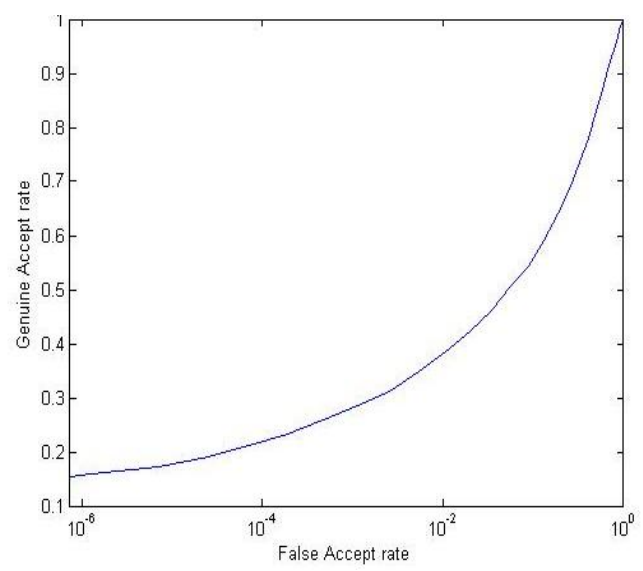

Fig. 3. ROC curve for Face Recognition.

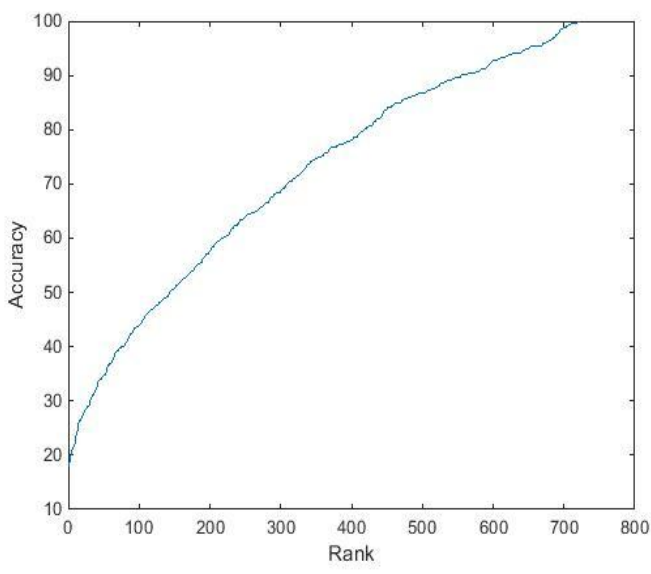

Fig. 4. CMC curve

\section{CONCLUSION}

In this research effort, the performance of face recognition was evaluated on a non-ideal FERET facial dataset. A CGT based patch selection model is explored to select only most important patches where each patch was considered as a player for recognition process. This selection model not only considered the individual importance of a patch but also considered its interactions with other patches based on Shapley value. An mLBP technique was applied to extract features from each patch. The game theoretic face recognition model not only maintained better accuracy but also it reduced the amount of image area required for performance evaluation.

\section{ACKNOWLEDGMENT}

This research is funded by Science and Technology Center: Bio/Computation Evolution in Action Consortium (BEACON).

\section{REFERENCES}

[1] J. Wright and G. Hua, "Implicit elastic matching with random projections for pose-variant face recognition," Computer Vision and Pattern Recognition, Florida, pp. 1502-1509, 2009.

[2] P. Dreuw, P. Steingrube, H. Hanselmann, and H. Ney, "SURF Face: face recognition under viewpoint consistency constraints," in Proc. the British Machine Vision Conference, London, 2009, pp. 1-7.

[3] J. R. Solar, R. Verschae, and M. Correa, "Recognition of faces in unconstrained environments: A comparative study," EURASIP Journal on Advances in Signal Processing, vol. pp. 1-20, 2009.
[4] D. Maturana, D. Mery, and Á. Soto, "Face Recognition with local binary patterns, spatial pyramid histograms and naive bayes nearest neighbor classification," in Proc. the SCCC International Conference of the Chilean Computer Science Society, Chile, 2009, pp. 125-132.

[5] T. Ahonen, A. Hadid, and M. Pietikinen, "Face description with local binary patterns: Application to face recognition," IEEE Transactions on Pattern Analysis and Machine Intelligence, vol. 28, no. 12, pp 2037-2041, Dec. 2006.

[6] T. Ojala, M. Pietikäinen, and T. Mäenpää, "Multiresolution gray-scale and rotation invariant texture classification with local binary pattern," IEEE Trans. on Pattern Analysis and Machine Intelligence, vol. 24, no. 7, pp. 971-987, 2002.

[7] Z. Guo, L. Zhang, and D. Zhang, "A completed modeling of local binary pattern operator for texture classification," IEEE Trans. on Image Processing, vol. 19, no. 6, pp. 1657-1663, June 2010.

[8] V. Ghosal, P. Tikmani, and P. Gupta, "Face classification using gabor wavelets and random forest," in Proc. 2009 Canadian Conference on Computer and Robot Vision, 2009, pp. 68-73.

[9] L. Shen, L. Bai, D. Bardsley, and Y. Wang, "Gabor feature selection for face recognition using improved adaboost learning," in Advances in Biometric Person Authentication, Springer Berlin Heidelberg, pp. $39-49,2005$.

[10] J. Huang, Y. Cai, and X. Xu, "A hybrid genetic algorithm for feature selection wrapper based on mutual information," Pattern Recognition Letters, vol. 28, no. 13, pp. 1825-1844, 2007.

[11] S. Lin, Z. Lee, S. Chen, and T. Tseng, "Parameter determination of support vector machine and feature selection using simulated annealing approach," Applied Soft Computing, vol. 8, no. 4, pp. 1505-1512, 2008

[12] S. P. Moustakidis and J. B. Theocharis, "Svm-fuzcoc: A novel svm-based feature selection method using a fuzzy complementary criterion," Pattern Recognition, vol. 43, no. 11, 2010, pp. 3712-3729.

[13] R. Xiao, W. Li, Y. Tian, and X. Tang, "Joint boosting feature selection for robust face recognition," in Proc. the Computer Vision and Pattern Recognition, 2006 IEEE Computer Society Conference, vol. 2, pp. 1415-1422, 2009.

[14] S. Kotsiantis, "Feature selection for machine learning classification problems: a recent overview," Artificial Intelligence Review, pp. 1-20, 2011.

[15] L. Shapley, "A value for n-person games," in Contributions to the Theory of Games H. Kuhn and A. Tucker, Eds., Princeton: Princeton University Press, vol. II, pp. 307-317, 2002.

[16] F. Ahmad, K. Roy, and K. Popplewell, "Multispectral iris recognition using patch based game theory," Image Analysis and Recognition, Lecture Notes in Computer Science, pp.112-119, 2014.

[17] S. Cohen, G. Dror, and E. Ruppin, "Feature selection via coalition game theory," Neural Computation, vol. 19, no. 7, pp. 1939-1961, 1939.

[18] S. Cohen , E. Ruppin, and G. Dror, "Feature selection based on the Shapley value," in Proc. 19th international joint conference on Artificial intelligence, Edinburgh, Scotland, pp. 665-670, July 30-August 05, 2005.

[19] P. Phillips, H. Wechslerb, J. Huangb, and P. Raussa, "The FERET database and evaluation procedure for face-recognition algorithms," Image and Vision Computing, vol. 16, no. 5, pp. 295-306, 1998.

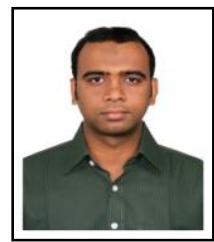

Foysal Ahmad is currently a MS student at the Department of Computer Science, North Carolina A\&T State University, North Carolina, U.S.A. He received his B.Sc. degree from Bangladesh University of Engineering and Technology (BUET), Dhaka Bangladesh. He also worked at Samsung R\&D Institute, Dhaka, Bangladesh as software engineer. His research interests include biometrics, computer vision, image processing, pattern recognition, and machine learning. He has published 2 journal and 3 conference articles.

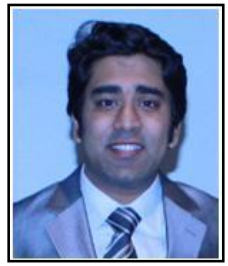

Kaushik Roy received his $\mathrm{PhD}$ from Concordia University, Montreal, QC, Canada in 2011 in computer science. He also completed his MS degree in computer science from the Concordia University in 2006 and B.Sc. degree in computer science from University of Rajshahi, Bangladesh in 2001.

Kaushik Roy is currently an assistant professor at the Department of Computer Science, and Assistant Director of the Center for Advanced Studies in Identity Sciences (CASIS), North Carolina A\&T State University, USA. Previously, he worked as a postdoctoral fellow in the Department of Electrical and Computer Engineering, University of Waterloo, ON, Canada 
during 2011-2012. He also taught at Rajshahi University of Engineering and Technology (RUET) as a lecturer of the Department of Computer Science and Engineering during 2001-2004.

Dr. Kaushik Roy is also the recipient of several fellowships and awards including the prestigious NSERC Visiting Fellowship, FQRNT B3 (Postdoctoral), NSERC (Doctoral) and FQRNT B2 (Doctoral). His research interests include biometrics, cyber identity, game theory, information fusion, computer vision, machine learning, and pattern recognition. He has published 1 book, 2 book chapters, 15 journal articles and over 40 conference articles.

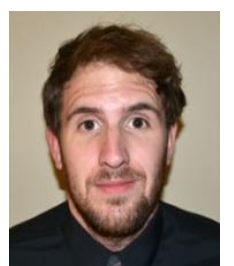

Brian O'Connor is an undergraduate student at North Carolina Agricultural and Technical State University, Greensboro, NC, USA, in the Computer Science Department. Brian is scheduled to graduate with his bachelor's degree in computer science in spring 2014. $\mathrm{He}$ is currently working as a research assistant at North Carolina A\&T and has been in his position for a year. His research interests include cyber identity, biometrics and machine learning.

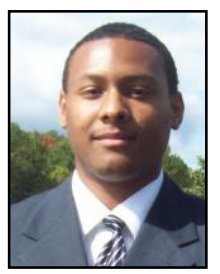

Joseph Shelton is a doctoral student at North Carolina Agricultural and Technical State University, Greensboro, NC, USA, in the Computer Science Department. Joseph obtained both his bachelor's degree and master's degree in computer science at North Carolina A\&T State University in 2010 and 2012.

$\mathrm{He}$ is currently working as a research assistant at North Carolina A\&T and has done so for the last three years. He has helped publish a book chapter in 'New Trends and Developments in Biometrics' titled 'Genetic and Evolutionary Biometrics' and has published over 20 articles in the field of biometrics and genetic \& evolutionary computations. Joseph's research interests include biometrics, cyber security and evolutionary computation.

Mr. Joseph Shelton has received an award for $1^{\text {st }}$ runner up for best student paper at the conference on systems engineering research (CSER 2012).

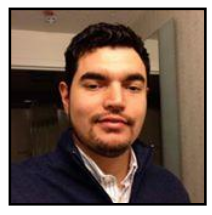

Pablo A. Arias is an undergraduate student at North Carolina Agricultural and Technical State University, Greensboro, NC, USA. Pablo is scheduled to graduate with his B.S. in computer science in spring 2014. He is currently working as a research assistant at North Carolina A\&T and has been in his position for a year. identity, and biometrics.

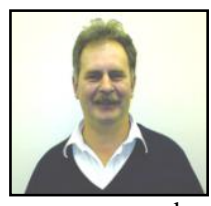

Albert Esterline is an associate professor of computer science at North Carolina A\&T State University. He has graduated over 70 MS students and led the Department's successful effort in establishing a PhD program. His research interests include formal methods, multiagent systems, the semantic web, situation awareness, and network science. Dr. Esterline is the author or co-author of over 100 articles in journals and conference proceedings and four book chapters. Dr. Esterline received a PhD in philosophy from the University of St. Andrews; he also received an MS in mathematics and a PhD in computer science from the University of Minnesota. Recent grants are for research on multiagent systems, sensor networks, machine learning, and ontologies. $\mathrm{He}$ frequently leverages his multidisciplinary background in his research.

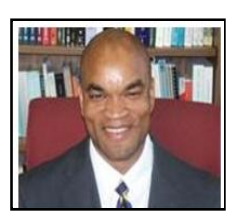

Gerry Vernon Dozier is a professor and the chair of the Computer Science Department at North Carolina A\&T State University. He earned his Ph.D. from North Carolina State University. He is the director of the Center for Advanced Studies in Identity Sciences (CASIS), as well as the PI for the Center for Cyber Defense (recognized by the National Security Agency and the Department of Homeland Security as a Center for academic excellence in information assurance education).

During Gerry's tenure as chair, the Department has seen an increase in extramural funding and research publications as well as the establishment of a Ph.D. program. He has also lead in the development of an undergraduate research program where approximately $20 \%$ of the undergraduate students are active participants in funded research projects. Under Gerry's leadership, the NSF Alliance for the Advancement of African American Researchers in Computing (A4RC, www.a4rc.org) experienced a threefold increase (from 6 to 20) in the number of participating universities. A4RC was effective in increasing the number of African-American recipients of advanced degrees in Computer Science.

Dr. Gerry Vernon Dozier has published over 130 conference and journal publications. He has served as an associate editor of the IEEE Transactions on Evolutionary Computation and the International Journal of Automation \& Soft Computing. Gerry is also a member of the editorial board for the International Journal of Intelligent Computing \& Cybernetics. His research interests include artificial and computational intelligence, genetic, evolutionary, and neural computing, biometrics, identity sciences, cyber identity, distributed constraint reasoning, artificial immune systems, machine learning and network intrusion detection. 